\title{
ON THE CURVATURE OF THE LEVEL LINES OF A HARMONIC FUNCTION
}

\author{
R. P. JERRARD AND L. A. RUBEL
}

Let $u(z)$ be a harmonic function in some simply connected region $W$ in the complex plane, with grad $u \neq 0$ in $W$. Let $\Gamma\left(z_{0}\right)$ $=\left\{z: u(z)=u\left(z_{0}\right)\right\}$ be the level curve of $u$ that passes through $z_{0}$, and let $K\left(z_{0}\right)$ denote the curvature of $\Gamma\left(z_{0}\right)$ at the point $z_{0}$.

We study some of the simple properties of $K(z)$. Briefly, $\log |K(z)|$ is superharmonic so that $|K(z)|$ satisfies the minimum property. An example shows that $|K(z)|$ need not satisfy the maximum property.

First, we obtain a useful formula for $K(z)$ by selecting a function $w(z)=u(z)+i v(z)$, holomorphic in $W$, whose real part is $u$. Then

$$
K=\left|w^{\prime}\right| \operatorname{Re}\left\{w^{\prime \prime} /\left(w^{\prime}\right)^{2}\right\} .
$$

A related formula (see, for example, Pólya and Szegö, Aufgaben und Lehrsätze aus der Analysis, Vol. 1, p. 105) has been used extensively in function theory.

To prove (1), we take $K$ as $d \theta / d s$, where $s$ is arc length along $\Gamma\left(z_{0}\right)$, and $\theta$ is the angle of inclination of the vector normal to $\Gamma\left(z_{0}\right)$. Hence

$$
\begin{aligned}
\theta & =-\arg w^{\prime}[z(s)], \\
\frac{d \theta}{d s} & =\frac{d}{d s}\left[\operatorname{Im}\left(\log w^{\prime}\right)\right]=\operatorname{Im}\left[\frac{w^{\prime \prime}}{w^{\prime}} \frac{d z}{d s}\right],
\end{aligned}
$$

where primes denote differentiation with respect to $z$. But along a level curve of $u$,

$$
\frac{d z}{d s}=\frac{d z}{d w} \frac{d w}{d s}=\frac{i}{w^{\prime}} \frac{d v}{d s}= \pm i \frac{\left|w^{\prime}\right|}{w^{\prime}},
$$

and (1) follows from (3) and (4) except for the choice of sign, which is a matter of definition.

Theorem. $\log |K(z)|$ is superharmonic where $K(z) \neq 0$.

Proof. Since $\log \left|w^{\prime}(z)\right|$ is harmonic, it follows from (1) that $\Delta \log |K(z)|=\Delta \log |U(z)|$, where $U=\operatorname{Re}\left(w^{\prime \prime} /\left(w^{\prime}\right)^{2}\right)$ is harmonic. An easy calculation then shows that

$$
\Delta \log |K(z)|=-|\operatorname{grad} U|^{2} / U^{2} \leq 0 .
$$

Received by the editors February 6, 1962. 
This formula is invalid only when $\left|w^{\prime}\right|=0$ or when $U=0$, and both of these cases have been excluded by hypothesis. q.e.d.

CoROllary. $|K(z)|$ has the minimum property. More precisely,

$$
\inf _{z \in W}|K(z)|=\inf _{t \in \partial W} \liminf _{z \rightarrow t}|K(z)| \text {. }
$$

Proof. Since $\log |K(z)|$ is superharmonic, $|K(z)|$ may not have a local minimum at $z_{0}$ unless $K\left(z_{0}\right)=0$. But if $K\left(z_{0}\right)=0$, then $U\left(z_{0}\right)=0$, since it has been required, to begin with, that $\left|w^{\prime}\right|=|\operatorname{grad} u| \neq 0$ in $W$. Thus, the locus $K(z)=0$ is itself the level line, $U(z)=0$ of the harmonic function $U$, and by the maximum principle for harmonic functions, this locus extends to the boundary of $W$.

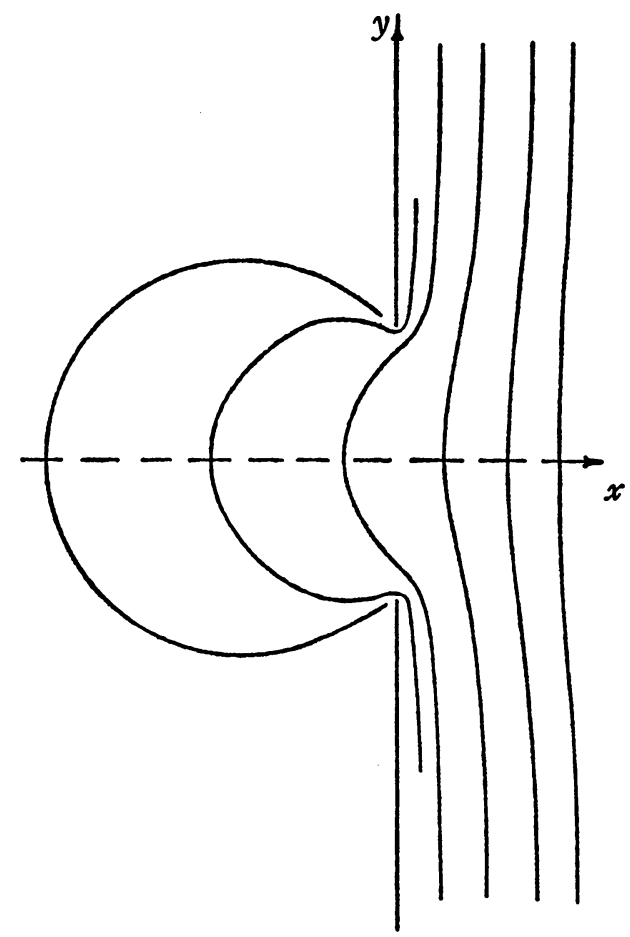

EXAmple. A sketch of the level lines of

$$
u(z)=\operatorname{Re}\left\{z+\left(z^{2}+1\right)^{1 / 2}\right\}
$$

makes it plausible that $|K(z)|$ can, on the other hand, have a strict maximum at an interior point of $W$, where in this case $W$ is the $z$ plane with all points of the $y$-axis except those for which $|y|<1$ re- 
moved. A hydrodynamical interpretation gives these lines as the stream lines of an ideal flow from North to South in the right half plane after the portion of the $y$-axis from $y=-1$ to $y=+1$ is removed. The sketch indicates that the stream lines are very flat and nearly vertical near the $x$-axis when $|x|$ is large. This suggests the curvature has a strict local maximum somewhere on the real axis.

For an analytic proof that this is, in fact, the case, we write

$$
\begin{gathered}
w=z+\left(z^{2}+1\right)^{1 / 2}=u+i v, \\
z=\frac{1}{2}\left(w-\frac{1}{w}\right), \\
\left(z^{2}+1\right)^{1 / 2}=\frac{1}{2}\left(w+\frac{1}{w}\right) \\
w^{\prime}=w\left(z^{2}+1\right)^{-1 / 2} \\
w^{\prime \prime}=\left(z^{2}+1\right)^{-3 / 2} .
\end{gathered}
$$

From (1) and (7)-(11), an expression for $K$ in terms of $u$ and $v$ is readily derived.

$$
\begin{aligned}
K & =\left|w^{\prime}\right| \operatorname{Re} \frac{w^{\prime \prime}}{\left(w^{\prime}\right)^{2}}=\frac{4}{\left|w^{2}+1\right|^{3}} \operatorname{Re}\left(w^{3}+w\right) \\
& =\frac{4 u\left(u^{2}-3 v^{2}+1\right)}{\left[\left(u^{2}-v^{2}+1\right)^{2}+4 u^{2} v^{2}\right]^{3 / 2}} .
\end{aligned}
$$

We now show that the anticipated maximum occurs at $p_{0}=\left(u_{0}, v_{0}\right)$ $=(1 / \sqrt{3}, 0)$, which, in the $z$-plane, is the point $z_{0}=-1 / \sqrt{3}$. For,

(13) $\frac{\partial}{\partial v} \log K=-6 v\left\{\frac{\left(u^{2}+v^{2}-1\right)\left(u^{2}-3 v^{2}+1\right)+\left(u^{2}-v^{2}+1\right)^{2}+4 u^{2} v^{2}}{\left(u^{2}-3 v^{2}+1\right)\left(\left(u^{2}-v^{2}+1\right)^{2}+4 u^{2} v^{2}\right)}\right\}$.

Since the expression in brackets is positive at $\left(u_{0}, v_{0}\right)$, it follows that

$$
\operatorname{sgn} \frac{\partial K}{\partial v}=-\operatorname{sgn}(v)
$$

throughout some small neighborhood $W^{*}$ of $p_{0}$, which is the image of some neighborhood $W^{\prime}$ of $z_{0}$. Hence, for $(u, v) \in W^{*}$, we have $K(u, v)<K(u, 0)$ unless $v=0$. Furthermore, $K(u, 0)=u\left(u^{2}+1\right)^{-2}$, which has a strict maximum at $u=u_{0}$. Thus, for $(u, v) \in W^{*}, K(u, v)$ $\leq K\left(u, v_{0}\right) \leq K\left(u_{0}, v_{0}\right)$ with equality only if $(u, v)=\left(u_{0}, v_{0}\right)$, and the assertion is proved.

We conclude with some remarks. If $f(z)$ is a holomorphic (and non- 
vanishing) function inside the unit disc, then it is easy to construct a holomorphic function $w(z)=u(z)+i v(z)$ for which $f(z)=-1 / w^{\prime}(z)$. Then from (1) we have $K(z)=\operatorname{Re}\left\{f^{\prime}(z) /|f(z)|\right\}$. It then follows that

$$
\inf _{|z|<1} \operatorname{Re}\left\{f^{\prime}(z) /|f(z)|\right\} \leq 1,
$$

since otherwise the level lines of $u$ would all have curvature exceeding 1 in the unit disc. The following argument shows that this is impossible. Suppose $K>1$ for all level curves of $u$ in $|z|<1$. Then we can find a closed disc $D:|z| \leq 1-\epsilon$ in which $K>1 /(1-\epsilon)$. By the strong maximum principle the level curves where $u$ attains its maximum and minimum, say $u=M$ and $u=m$, must be tangent to the circle $C:|z|$ $=1-\epsilon$. At least one of them must be an inner tangent, for if not the sign of $K$ must change as one moves from one such tangent point to another along an arc consisting of subarcs of the level curves $u=$ constant and their orthogonal trajectories. But if the curve $u=M$ is an inner tangent to $C$ at $P$, then near $P$ there are points in the interior of $D$ where $u=M$, and this violates the strong maximum principle. The extremal function $f(z)=-(z-1)^{2}$ shows that (15) is best possible. There are other proofs of (15).

Another curvature formula for harmonic functions can be obtained from (1). We have

$$
K=\left|w^{\prime}\right| \operatorname{Re}\left[\frac{w^{\prime \prime}}{w^{\prime}} \frac{d z}{d w}\right]=\left|w^{\prime}\right| \operatorname{Re} \frac{d}{d w} \log w^{\prime} .
$$

If we take the derivative in the direction normal to the level lines of the harmonic function $u$, then $d v=0, d u=d w$, and

$$
K=\left|w^{\prime}\right| \frac{d}{d u} \operatorname{Re} \log w^{\prime}=\left|w^{\prime}\right| \frac{d}{d u} \log \left|w^{\prime}\right|=\frac{d\left|w^{\prime}\right|}{d u} .
$$

Further, if the normal directional derivative is denoted by $d / d n$, then $d u / d n=\left|w^{\prime}\right|=|\operatorname{grad} u|$, and we have the formula

$$
K=\frac{d}{d n} \log |\operatorname{grad} u| \text {. }
$$

It is easy to integrate $K$ along the orthogonal trajectories to the level curves of $u$. For example, if such an orthogonal trajectory forms a closed curve, the mean of $K$ along that curve must be zero.

UNIVERSITY OF ILLINOIS 